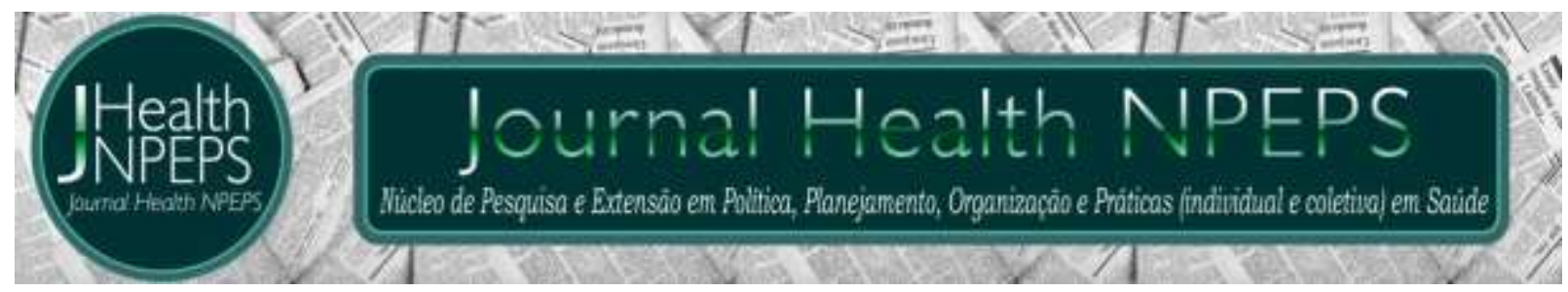

http://dx.doi.org/10.30681/252610103361

ARTIGO ORIGINAL

\title{
Avaliação da situação nutricional e dietética de idosos hospitalizados
}

\author{
Assessment of nutritional and dietary status of hospitalized elderly
}

\section{Evaluación de la situación dietética y nutricional de los ancianos hospitalizados}

\section{Tatiane dos Reis Aquino ${ }^{1}$, Ionara Almeida Mairink², Samara Cardoso de Jesus ${ }^{3}$, Gina Tanúria Guerra da $\mathrm{Cruz}^{4}$, Letícia Josyane Ferreira Soares ${ }^{5}$, Aline Fernandes de Souza ${ }^{6}$, Niadena Rodrigues Trindade Martins ${ }^{7}$, Vanessa Santos Silva ${ }^{8}$, Fernanda Gabriele Santos Rocha9, Rodrigo Pereira Prates ${ }^{10}$, Ana Maria de Jesus Teixeira Alves ${ }^{11}$, Paula Karoline Soares Farias ${ }^{12}$}

\section{RESUMO}

Objetivo: avaliar a situação nutricional e dietética de idosos hospitalizados. Método: pesquisa do tipo descritiva, exploratória, transversal e quantitativa. Foi realizada com 44 idosos hospitalizados, de ambos os sexos, atendidos em um hospital público da cidade

\footnotetext{
${ }^{1}$ Nutricionista. Faculdade de Saúde Ibituruna - FASI. Montes Claros, Minas Gerais, Brasil. E-mail: tattinutri2010@gmail.com ORCID ID: https://orcid.org/0000-0002-8206-7721

${ }^{2}$ Acadêmica de Nutrição. Faculdade de Saúde Ibituruna - FASI. Montes Claros, Minas Gerais, Brasil. E-mail: ionaramairink@hotmail.com ORCID ID: https://orcid.org/0000-0001-7835-2581

${ }^{3}$ Acadêmica de Nutrição. Faculdade de Saúde Ibituruna - FASI. Montes Claros, Minas Gerais, Brasil. E-mail: samaracardoso700@gmail.com ORCID ID: https://orcid.org/0000-0001-8076-2766

${ }^{4}$ Médica. Especialização. Docente. Faculdades Unidas do Norte de Minas - FUNORTE. Montes Claros, Minas Gerais, Brasil. E-mail: gginatanuria@yahoo.com.br ORCID ID: https://orcid.org/0000-0003-2754-0130

${ }^{5}$ Nutricionista. Mestre. Docente. Universidade Federal de Minas Gerais - UFMG. Belo Horizonte, Minas Gerais, Brasil. E-mail: leticiasoares.nutricionista@yahoo.com.br ORCID ID: https://orcid.org/0000-0002-2427-6453

${ }^{6}$ Farmacêutica. Mestre. Universidade Estadual de Montes Claros - Unimontes. Montes Claros, Minas Gerais, Brasil. Email: alineffarma@gmail.com ORCID ID: https://orcid.org/0000-0001-7201-6042

${ }^{7}$ Nutricionista. Mestranda. Universidade Federal de Minas Gerais - UFMG. Belo Horizonte, Minas Gerais, Brasil. E-mail: niadena@hotmail.com ORCID ID: https://orcid.org/0000-0001-9754-8379

${ }^{8}$ Nutricionista. Especialista. Nutricionista hospitalar. Faculdade de Saúde Ibituruna - FASI. Montes Claros, Minas Gerais, Brasil. E-mail: vanaurora@gmail.com ORCID ID: https://orcid.org/0000-0002-0520-5107

${ }^{9}$ Acadêmica de Nutrição. Faculdade de Saúde Ibituruna - FASI. Montes Claros, Minas Gerais, Brasil. E-mail: fernandagau@hotmail.com ORCID ID: https://orcid.org/0000-0002-4959-663X

${ }^{10}$ Nutricionista. Mestre. Universidade Federal dos Vales do Jequitinhonha e Mucuri - UFVJM. Diamantina, Minas Gerais, Brasil. E-mail: rodrigo pprates@yahoo.com.br ORCID ID: https://orcid.org/0000-0003-4472-1857

${ }^{11}$ Enfermeira. Mestranda em ciências da saúde. Enfermeira do SAMU. Universidade Estadual de Montes Claros Unimontes. Montes Claros, Minas Gerais, Brasil. E-mail: anamalves2004@yahoo.com.br ORCID ID: https://orcid.org/0000-0001-5206-6173

${ }^{12}$ Nutricionaista. Doutoranda. Nutricionista na Secretária Municipal de Educação de Montes Claros. Universidade Estadual de Montes Claros - Unimontes. Montes Claros, Minas Gerais, Brasil. E-mail: paulak.soares@hotmail.com ORCID ID: https://orcid.org/0000-0003-0529-2754. Autora principal - Endereço para correspondência: Universidade Estadual de Montes Claros - Unimontes, Av. Prof. Rui Braga, S/N - Vila Mauriceia, CEP: 39401-089, - Montes Claros, MG, Brasil.
}

Este artigo está licenciado sob forma de uma licença Creative Commons Atribuição 4.0 Internacional, que permite uso irrestrito, distribuição e reprodução em qualquer meio, desde que a publicação original seja corretamente citada. 
de Bocaiúva, Minas Gerais, Brasil. Para avaliar a situação nutricional e dietética dos idosos hospitalizados utilizou-se a mini avaliação nutricional. Resultados: verifica-se que $55 \%$ dos idosos apresentaram-se com baixo peso, com maior quantitativo no sexo masculino. Observou-se prevalência de hipertensão, diabetes e insuficiência cardíaca congestiva. Entre os participantes, $25 \%$ informaram que possuem alguma restrição na motilidade. Identificou que mais de $60 \%$ dos idosos utilizam acima de três medicamentos e bebem menos de cinco copos de água por dia. Conclusão: a realização de pesquisas sobre o estado nutricional de idosos e a associação de fatores que contribuem para agravar o quadro de desnutrição é fundamental para definir e acompanhar a qualidade de vida, bem como sustentar projetos terapêuticos.

Descritores: Consumo de Alimentos; Estado Nutricional; Nutrição do Idoso; Saúde do idoso Institucionalizado.

\section{ABSTRACT}

Objective: to evaluate the nutritional and dietary situation of hospitalized elderly. Method: descriptive, exploratory, cross-sectional and quantitative research. It was carried out with 44 elderly inpatients, both genders, treated in a public hospital in the city of Bocaiúva, Minas Gerais, Brazil. To assess the nutritional and dietary status of the hospitalized elderly, the mini nutritional assessment was used. Results: $55 \%$ of the elderly were underweight, with higher numbers in males. Prevalence of hypertension, diabetes and congestive heart failure were observed. Among the participants, 25\% reported to have some restriction on motility. Over $60 \%$ of the elderly were found to use more than three medications and drink less than five glasses of water per day. Conclusion: conducting research on the nutritional status of the elderly and the association of factors that contribute to aggravate malnutrition is fundamental to define and monitor quality of life, as well as to sustain therapeutic projects.

Descriptors: Food Consumption; Nutritional Status; Elderly Nutrition; Health of Institutionalized Elderly.

\section{RESUMEN}

Objetivo: valorar el estado nutricional y dietética de ancianos hospitalizados. Método: estudio descriptivo, exploratorio, transversal y el análisis cuantitativo. Se llevó a cabo con 44 ancianos hospitalizados, de ambos sexos, atendidos en un hospital público de la ciudad de Bocaiúva, Minas Gerais, Brasil. Para evaluar la situación dietética y nutricional de los ancianos hospitalizados utilizó el mini nutricional assessment. Resultados: el 55\% de los ancianos tenían bajo peso, con un mayor número de hombres. Se observó prevalencia de hipertensión, diabetes e insuficiencia cardíaca congestiva. Entre los participantes, el $25 \%$ informó que tienen alguna restricción en la motilidad. Descubrió que más del $60 \%$ de las personas mayores usan más de tres medicamentos y beben menos de cinco vasos de agua al día. Conclusión: realizar investigaciones sobre el estado nutricional de los ancianos y la asociación de factores que contribuyen a agravar la desnutrición es fundamental para definir y controlar la calidad de vida, así como para sostener proyectos terapéuticos.

Descriptores: Consumo de Alimentos; Estado Nutricional; Nutricion del Anciano; Salud del Anciano Institucionalizado.

\section{INTRODUÇÃO}

Envelhecer é uma condição natural e inconversível da população ${ }^{1}$ e acontece pela diminuição das taxas de fecundidade, mortalidade e também pela melhora da expectativa de vida². A população idosa no mundo é de 
aproximadamente 900 milhões e estimativas elevam esta estatística para cerca de 2 bilhões em $2050^{3}$. O país tem hoje cerca de 20 milhões de idosos, com previsão para 2025, de ultrapassar 32 milhões $^{4}$, e classificado como o sexto país do mundo com o número de pessoas idosas ${ }^{3}$.

O Brasil vive um processo de transição epidemiológica ${ }^{5}$, pois envelhece de forma acelerada6 ${ }^{6}$, doenças crônicas características do processo de senescência são evidentes no cenário da saúde pública ${ }^{5}$, visto que esta população pode apresentar síndromes geriátricas que acarretam uma diminuição na capacidade funcional ${ }^{7}$. São percebidas também modificações morfológicas, fisiológicas, metabólicas, imunológicas ${ }^{8}$ e maior dependência concernente às atividades cotidianas ${ }^{7}$.

Todavia as cidades e o sistema de saúde mostram-se frágeis por não gerar qualidade de vida a esta população, caracterizada como grupo vulnerável que mais recorre ao sistema de saúde 9 . Esta população é altamente acometida por doenças crônicas não transmissíveis que eleva a estada intrahospitalar, retardamento da recuperação clínica e aumento da incidência de reinternações ${ }^{10}$.

A avaliação nutricional é uma ferramenta eficaz que permite caracterizar e classificar Journal Health NPEPS. 2019 jul-dez; 4(2):268-279. nutricionalmente uma determinada população ${ }^{11}$. Visto que a nutrição modula o processo de envelhecimento no aparecimento como na etiologia das doenças associadas à idade ${ }^{12}$ e nesta conjuntura, o estado nutricional é imprescindível tanto na recuperação quanto no tratamento clínico deste idoso $^{13}$.

Em pacientes hospitalizados a desnutrição é algo comum e frequente, havendo necessidade de avaliações nutricionais periódicas nestes pacientes, para que seja possível introduzir a terapia nutricional correta. 0 adequado estado nutricional é importante para a recuperação e tratamento clínico de enfermos, principalmente em pacientes hospitalizados por muito tempo ${ }^{14}$, especialmente no grupo dos idosos, além da desnutrição, verifica-se a maior prevalência das doenças crônicas nãotransmissíveis associadas ao diagnóstico do paciente ${ }^{15}$.

Observa-se a importância do profissional nutricionista no ambiente hospitalar, uma vez que este é o responsável pelo fornecimento e adequação da dieta para o paciente ${ }^{16}$, e neste contexto o nutricionista hospitalar irá realizar a avaliação nutricional, adequar a dieta à necessidade patológica do paciente, acompanhar a ingestão das refeições servidas ${ }^{17}$.

Detectar precocemente uma 
deficiência nutricional que resulte da escassez de nutrientes em um organismo propicia o restabelecimento deste estado nutricional e transforma-o em um agente ativo na promoção da redução da morbimortalidade, das complicações operatórias e também na prevenção e na procrastinação da progressão da desnutrição ${ }^{18}$ e entre idosos essa é uma preocupação maior. Assim, este trabalho teve como objetivo avaliar a situação nutricional e dietética de idosos hospitalizados.

\section{MÉTODO}

Trata-se de um estudo descritivo, exploratório, transversal e quantitativo, envolvendo 44 idosos hospitalizados com ou sem patologias, de ambos os sexos com idade igual ou superior a 60 anos, atendidos em um hospital público da cidade de Bocaiúva MG, Brasil.

Esta cidade está localizada no norte de Minas Gerais, apresentando Índice de Desenvolvimento Humano (IDHM) de 0,700, o que situa o município na faixa de IDHM alto (entre 0,700 e 0,799). A dimensão que mais contribui para o IDHM do município foi a longevidade, com índice de 0,822, seguida de renda e educação. Conforme o último censo demográfico, a cidade apresenta 4.391 pessoas com idade acima de 60 anos, sendo que entre 2000 e 2010, a taxa de envelhecimento passou de $4,88 \%$ para $6,80 \%^{4}$.

A coleta de dados ocorreu no período de setembro a dezembro de 2017, por meio das pesquisadoras devidamente treinadas, com 0 preenchimento do instrumento e realização da entrevista/avaliação. Os dados foram coletados no próprio leito do paciente, através de uma entrevista padronizada, da Mini Avaliação Nutricional (MAN) ${ }^{19,20}$ para avaliar a situação dietética dos idosos hospitalizados e a avaliação antropométrica completa ${ }^{21}$.

$\mathrm{Na}$ avaliação antropométrica foi utilizada uma balança digital para mensuração do peso, estadiômetro para aferição da altura, e foi realizado o diagnóstico nutricional pelo Índice de Massa Corporal (IMC).

Os dados foram analisados considerando pontes de cortes específicos para idosos ${ }^{22}$, conforme demonstrado no quadro 1. 
Quadro 1 - Pontos de corte do IMC estabelecidos para idosos e o respectivo diagnóstico nutricional.

Menor ou igual a $22 \mathrm{Kg} / \mathrm{m}^{2}$

Maior que $22 \mathrm{Kg} / \mathrm{m}^{2}$ e menor que $27 \mathrm{Kg} / \mathrm{m}^{2}$ Maior ou igual que $27 \mathrm{Kg} / \mathrm{m}^{2}$ Sobrepeso

Fonte: Lipschitz ${ }^{22}$.

Os dados foram analisados por estatística descritiva simples.

Primeiramente, os dados foram tabulados no programa Excel 2010, em seguida transportados para o software Bioestat $3.0^{23}$ e por fim apresentação de tabelas, com números absolutos e relativos.

A pesquisa foi realizada respeitando os preceitos éticos propostos pelo Conselho Nacional de Saúde (CNS), conforme a Resolução 466/2012, e aprovado por Comitê de Ética e Pesquisa com Seres Humanos (CEP) sob o parecer $n^{\circ} \quad 2.216 .425$ e 0 CAAE $n^{\circ}$ 69775917.0.0000.5141. Todos os participantes do estudo assinaram 0
Termo de Consentimento Livre e Esclarecido (TCLE).

\section{RESULTADOS}

Participaram da pesquisa 44 idosos hospitalizados da cidade de Bocaiúva-MG. Dentre os participantes, verifica-se a prevalência de $54,5 \%(n=24)$ do sexo feminino. Observa-se que o Índice de Massa Corporal (IMC) apresentou-se adequado em 54,1\% $(\mathrm{n}=14)$ para o sexo feminino e em $55 \%$ $(n=11)$ para baixo peso no sexo masculino, conforme demonstrado na Tabela 1.

Tabela 1 - Perfil antropométrico dos idosos hospitalizados. Setembro a dezembro de 2017. Bocaiuva MG, Brasil.

\begin{tabular}{ccccc} 
Variável & $\begin{array}{c}\text { Feminino } \\
(\mathbf{n = 2 4 )}\end{array}$ & $\%$ & $\begin{array}{c}\text { Masculino } \\
(\mathbf{n}=\mathbf{2 0})\end{array}$ & $\%$ \\
IMC & & & & \\
Baixo peso & 07 & 33,4 & 11 & 55 \\
Adequado & 14 & 54,1 & 07 & 35 \\
Sobrepeso & 03 & 12,5 & 02 & 10 \\
\hline
\end{tabular}

Tabela 2 - Prevalência das patologias mais comuns dos idosos hospitalizados. Setembro a dezembro de 2017. Bocaiuva - MG, Brasil.

\begin{tabular}{lcccc}
\hline \multicolumn{1}{c}{ Patologias } & Feminino $(\mathbf{n}=\mathbf{2 4})$ & $\%$ & Masculino $(\mathbf{n}=\mathbf{2 0})$ & $\%$ \\
Hipertensão & 08 & 33,3 & 09 & 45 \\
Diabetes & 04 & 16,6 & 05 & 25 \\
Artrite Crônica & 01 & 4,2 & 00 & 0,0 \\
Dislipidemia & 01 & 4,2 & 00 & 0,0 \\
Osteoporose & 02 & 8,3 & 00 & 0,0 \\
Insuficiência Cardíaca Congestiva & 04 & 16,6 & 02 & 10 \\
Cálculo Renal & 01 & 4,2 & 00 & 0,0 \\
Hipertireoidismo & 01 & 4,2 & 00 & 0,0
\end{tabular}




\begin{tabular}{llllc} 
Demência ligeira & 01 & 4,2 & 00 & 0,0 \\
Câncer & 01 & 4,2 & 00 & 0,0 \\
Pneumonia & 00 & 0,0 & 02 & 10 \\
Gota & 00 & 0,0 & 01 & 5 \\
Colesterol Alto & 00 & 0,0 & 01 & 5 \\
\hline
\end{tabular}

Observa-se que há uma prevalência de hipertensão, diabetes e Insuficiência Cardíaca Congestiva (ICC) entre as patologias mais comuns entre os idosos, sendo que hipertensão e diabetes foram mais prevalentes no sexo masculino com $45 \%(n=9)$ e $25 \%(n=5)$, respectivamente, e a ICC no sexo feminino com 16,6\% ( $n=4)$ (Tabela 2).

Quanto à análise do consumo alimentar e a qualidade de vida, verificou-se que os idosos apresentaram redução moderada da ingestão alimentar, em especial no sexo feminino em $54,2 \%(n=13)$. A perda de peso foi afirmada pelos participantes nos últimos três meses. Quanto a motilidade, as idosas apresentam-se quase que $96 \%$ $(\mathrm{n}=23)$ sem nenhum comprometimento motor, enquanto que $25 \% \quad(n=5)$ dos idosos informaram que tem alguma restrição na motilidade.

A maioria dos entrevistados não apresentam problemas neurológicos e não foram submetidos a nenhum procedimento cirúrgico. Mais de $60 \%$ dos participantes utilizam acima de três medicamentos, conseguem realizar mais de três refeições e bebem menos de cinco copos de água por dia. Quando comparados à saúde de outros idosos, verifica-se que a maioria dos idosos não soube responder como está à própria saúde (Tabela 3).

Tabela 3 - Consumo alimentar e qualidade de vida dos idosos hospitalizados. Setembro a dezembro de 2017. Bocaiuva - MG, Brasil.

$\begin{array}{ccc}\text { Feminino } & \text { Masculino } \\ (n=24) & \% & (n=20)\end{array}$

A) Nos últimos três meses houve diminuição da ingestão alimentar devido à perda de apetite, problemas digestivos ou dificuldade para mastigar ou deglutir?

$0=$ diminuição grave da ingesta

$1=$ diminuição moderada da ingesta

2= sem diminuição da ingesta

$01 \quad 4,2 \quad 03 \quad 15$

$13 \quad 54,2 \quad 09$

$41,7 \quad 08$

B) Perda de peso nos últimos 3 meses.

$0=$ não sabe informar

$1=$ Menos de 1 quilo 10

$2=$ entre um e três quilos

$0,0 \quad 01$

$25 \quad 04$

00

29,2

04
08

45,8

$3=$ sem perda de peso

11

$\begin{array}{lll}07 & 29,2 & 08 \\ 11 & 45,8 & 07\end{array}$

C) Mobilidade.

$0=$ restrito ao leito ou a cadeira de roda

$1=$ deambula, mas não é capaz de sair de casa

00

$0,0 \quad 00$

$2=$ normal

05

$95,8 \quad 15$

40

D) Passou por algum estresse psicológico ou doença aguda nos últimos três meses?

$0=\operatorname{sim}$
$2=$ não

E) Problemas neuropsicológicos.

$0=$ demência ou depressões graves

02

22

8,3

01

$91,7 \quad 19$

5

00

$0,0 \quad 00$

0,0 
$1=$ demência Ligeira

$\begin{array}{llll}01 & 4,2 & 00 & 0,0\end{array}$

$2=$ sem problemas psicológicos

23

F) 0 doente vive na sua própria casa?

$1=\operatorname{sim}$

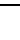

$0=$ não

22
02

H) Utiliza mais de três medicamentos por dia.

$0=\operatorname{sim}$

$1=$ não

16

100

I) Lesão de pele ou escaras.

$0=\operatorname{sim}$

08

$\begin{array}{lll}22 & 91,7 & 19\end{array}$

95

$1=$ não

01

23

8,3

5

J) Quantas refeições faz por dia?

$0=$ uma refeição

$1=$ duas refeições

00

04

20

66,7

01

12

$33,3 \quad 08$

60

2= Mais de duas refeições

83,3

$4,2 \quad 02$

$95,83 \quad 18$

10

90

L) 0 doente consome pelo menos uma porção diária de leite ou derivados (leite, queijo, iogurte)? Duas ou mais porções semanais de legumes ou ovos? carne, peixe ou aves todos os dias?
$0.0=$ nenhuma ou duas respostas "sim"
02
8,3
$0.5=$ duas respostas "sim"
08
33,3
$02 \quad 10$

0,0

00

0,0

$1.0=$ três respostas "sim"

$14 \quad 58,3 \quad 08$

M) 0 doente consome duas ou mais porções diárias de fruta ou produtos hortícolas?

$\begin{array}{lrrrr}0=\text { não } & 07 & 29,2 & 06 & 30 \\ 1=\operatorname{sim} & 17 & 70,8 & 14 & 70\end{array}$

N) Quantos copos de líquidos (água, suco, café, chá, leite) o doe

$0.0=$ menos de três copos

$0.5=$ três a cinco copos

$17 \quad 70,8 \quad 14$

$1.0=$ mais de cinco copos

0) Modo de se alimentar.

$\begin{array}{llll}05 & 20,8 & 03 & 15\end{array}$

$\begin{array}{llll}08 & 45,8 & 10 & 50\end{array}$

$0=$ não é capaz de se alimentar sozinho

$1=$ alimenta-se sozinho, porém com dificuldade

11

$33,3 \quad 07$

50

2= alimenta-se sozinho sem dificuldade

$\begin{array}{llll}00 & 0,0 & 00 & 0,0\end{array}$

P) 0 doente acredita ter algum problema nutricional?

$0=$ acredita estar desnutrido

$03 \quad 12,5 \quad 03$

$\begin{array}{llll}21 & 87,5 & 17 & 85\end{array}$

15

$1=$ não sabe dizer

$\begin{array}{llll}05 & 20,8 & 02 & 10\end{array}$

$\begin{array}{llll}15 & 62,5 & 14 & 70\end{array}$

$2=$ acredita não ter problema nutricional

04

$16,7 \quad 04$

10
70

20

Q) Em comparação com as outras pessoas da mesma idade, como considera o doente a sua própria saúde?
$0.0=$ pior
$0.5=$ não sabe
$1.0=$ igual
$2.0=$ melhor

01

19

4,2

79,2

01

5

02

$8,3 \quad 01$

$8,3 \quad 03$

75

02

\section{DISCUSSÃO}

0 envelhecimento desperta cada vez mais interesse, isso acontece por que os pacientes idosos apresentam peculiaridades clínicas e maior encaminhamento de recursos médicos e financeiros para estes ${ }^{24}$. Com o envelhecimento se tem o benefício de uma maior longevidade, mas com isso há um aumento de morbimortalidade, que acontece por se ter um maior número de doenças crônico-degenerativas nesta faixa etária. A preocupação com as condições de saúde de idosos tem ocasionado o aumento de muitos estudos sobre 0 envelhecimento humano ${ }^{2}$.

0 estado nutricional adequado é o reflexo do equilíbrio entre a ingestão balanceada de alimentos e o 
consumo de energia necessária para manter as funções diárias do organismo ${ }^{25}$. Quando algum fator interfere em qualquer uma das etapas desse equilíbrio, os riscos do indivíduo desenvolver desnutrição são eminentes, em especial nos idosos ${ }^{26}$.

Sabe-se que na prática clínica em nutrição realiza-se a aferição de diversas variáveis antropométricas a fim de se obter um diagnóstico nutricional mais preciso possível ${ }^{1}$. Contudo, ao se utilizar mais de uma variável, verifica-se 0 risco da aquisição de resultados divergentes, quando isso acontece é comum escolher como diagnóstico final aquele que aparece com uma frequência maior ${ }^{12}$.

A doença quase sempre, incide em modificações do metabolismo do paciente, não só pela própria enfermidade, como também pelo tratamento efetuado, caracterizando assim um desequilíbrio metabólico ${ }^{18}$. Os pacientes hospitalizados podem sofrer influência de variáveis de risco para o desenvolvimento da desnutrição, baixo peso ou excesso de peso, e muitas vezes inerentes à própria doença e à hospitalização ${ }^{14}$.
Fatores que colaboram para as principais causas de distúrbios nutricionais como alteração no olfato e no paladar (disgeusia) em consequência da diminuição das papilas na língua e das terminações nervosas, a ausência de dentição ou o uso de próteses totais e parciais ${ }^{27}$. Com a perda dos dentes, diminui-se a capacidade mastigatória e limita a seleção de alimentos. Neste contexto, os idosos sem dentição têm maior probabilidade de apresentar sinais de desnutrição do que aquelas com dentes ou com próteses totais adequadas $^{28}$.

A higiene oral precária e uso de próteses também afetam a capacidade percepção e sabor dos alimentos, como a atrofia de glândulas salivares, o tabagismo, o uso de um ou mais medicamentos, as doenças crônicas ou agudas resultando em xerostomia e disfagia, até mesmo a irregularidade intestinal ${ }^{25}$, podem comprometer a saúde do idoso.

Quando não tratadas rapidamente, as doenças crônicas não transmissíveis e o processo de desnutrição podem levar à morte prematura, perda de produtividade no local de trabalho e aposentadoria precoce, representando uma carga a 
mais para a sociedade, além da menor qualidade de vida não apenas para o indivíduo, mas também para a família, uma vez que os processos de internação tornam-se mais frequentes e demorados ${ }^{15}$.

Sendo assim, a pessoa idosa com ingestão elevada de um ou mais medicamentos, pode desenvolver aversão alimentar, associado aos efeitos colaterais, às doenças crônicas ou agudas, com restrição alimentar imposta ou voluntária, alcoolismo, incapacidade física ou mental, depressão, orçamento restrito, tabus alimentares e a solidão são alguns agravantes, para contribuir para a alimentação inadequada nesta fase da vida.

\section{CONCLUSÃO}

O baixo peso foi prevalente no sexo masculino e as patologias mais comuns entre os idosos foram hipertensão e diabetes. Verifica-se que os idosos relataram uma redução da ingestão alimentar, além do alto consumo de medicamentos. Percebese que a participação do idoso foi um limitante no estudo pela condição que o mesmo encontra-se ou dos familiares/responsáveis.
Observa-se a importância de realizar estudos que permitam analisar a ingestão alimentar, e comparar com o perfil nutricional desses pacientes, pois o Brasil está em processo de transição, com o crescimento desta faixa etária.

\section{REFERÊNCIAS}

1. Medeiros $P$, Lima RA, Sardinha AHL, Diniz DC, Aragão MAM. Aspectos nutricionais de idosos atendidos em um centro de saúde. Rev Pesq Saúde. 2014; 15(3):351-355.

2. Pimenta FB, Pinho L, Silveira MF, Botelho ACC. Fatores associados a doenças crônicas em idosos atendidos pela Estratégia de Saúde da Família. Ciênc Saúde Colet. 2015; 20(8):2489-2498.

3. OMS. Organização Mundial de Saúde. Relatório mundial de envelhecimento e saúde. Disponível em: http://sbgg.org.br/wpcontent/uploads/2015/10/OMS-

ENVELHECIMENTO-2015-port.pdf. Acesso em 20 set 2018.

4. IBGE. Instituto Brasileiro de Geografia e Estatística. Cidades. Disponível em: <http://cidades.ibge.gov.br/xtras/ perfil.php? Codmun=310730> . 
Acesso em 25 set 2018.

5. Stopa SR, César CLG, Segri NJ, Goldbaum M, Guimarães VMA, Alves MCGP, et al. Diabetes autor referido em idosos: comparação das prevalências e medidas de controle. Rev Saúde Pública. 2014; 48(4):554-562.

6. Azevedo AS, Oliveira DC, Soares PKD, Jones KM, Soares FBD, Silva $\mathrm{JN}$, et al. Perfil nutricional de pacientes adultos e idosos hospitalizados. Saúde pesqui. 2016; 9(1):25-29.

7. Hana K-M, Kob $\mathrm{Y}-\mathrm{H}$, Yoonb H-K, Hanb C, Hama B-J, Kimb Y-K. Relationship of depression, chronic disease, self-rated health, and gender with health care utilization among community-living elderly. J Affect Disord. 2018; 241:402-410.

8. Martins MV, Souza JD, Franco FS, Martinho KO, Tinôco ALA. Consumo alimentar de idosos e sua associação com 0 estado nutricional. HU Revista. 2016; 42(2):125-131.

9. Alves DSB, Barbosa MTS, Caffarena ER, Silva AS. Caracterização do envelhecimento populacional no município do Rio de Janeiro: contribuições para políticas públicas sustentáveis. Cad Saúde
Colet. 2016; 24(1):63-69.

10. Moreira RM, Teixeira RM, Novaes KO. Contribuições da atividade física na promoção da saúde, autonomia e independência de idosos. Rev Kairós. 2014; 17(1):201-217.

11. Fisberg RM, Marchioni DML, Castro $M A$, Verly Junior E, Araújo MC, Bezerra IN, et al. Inadequate nutrient intake among the Brazilian elderly: National Dietary Survey 2008-2009. Rev Saúde Pública. 2013; 47(1 Supl).

12. Tavares EL, Santos DM, Ferreira AA, Menezes MFG. Avaliação nutricional de idosos: desafios da atualidade. Rev Bras Geriatr Gerontol. 2015; 18(3):643-650.

13. Silva CFS, Dias CMSB. Violência Contra Idosos na Família: Motivações, Sentimentos e Necessidades do Agressor. Psicol Ciênc Prof. 2016; 36(3):637-652.

14. Silva DMM, Santos CM, Moreira MA. Perfil nutricional de pacientes internados em um hospital público de Recife-PE. Rev destaques acad. $2016 ; 8(3): 97-108$.

15. Pereira IFS, Spyrides MHC, Andrade LMB. Estado nutricional de idosos no Brasil: uma abordagem multinível. Cad Saúde 
Pública. 2016; 32(5):e00178814.

16. Amaral ACF, Rodrigues LA, Furlan RMMM, Vicente LCC, Motta AR. Speech-Language and Nutritional Sciences in hospital environment: analysis of terminology of food consistencies classification.

CoDAS. 2015; 27(6):541-549.

17. Mascarenhas RGT, Mainardes I, Carneiro CD. Eventos em hotelaria hospitalar: aspectos da gastronomia no fornecimento de cardápio para dietas restritivas. Rosa dos Ventos-Turismo e Hotelaria. 2016; 8(1):1-12.

18. Veras VS, Oliveira TR, Fortes RC, Salomon ALR. Prevalência de desnutrição ou riscos nutricional em pacientes cirúrgicos hospitalizados e correlação entre os métodos subjetivos e objetivos de avaliação do estado nutricional. Rev Bras Nutr Clín. 2016; 31(2):101-7.

19. Rubenstin LZ, Harker JO, Salvà A, Guigoz Y, Vellas B. Screening for under nutrition in geriatric practice: developing the Shortform Mini Nutritional Assessment (MNA-SF). J Gerontol A Biol Sci Med Sci. 2001; 56(6):M366-372.

20. Guigoz Y, Vellas BJ, Garry PJ. Mini Nutritional Assessment: a practical assessment tool for grading the nutritional state of elderly patients. Nutrition. 1999; 15(2):116-22.

21.Chumlea WMC, Roche AF, Steinbaugh ML. Estimating stature from knee height for persons 60 to 90 years of age. J Am Geriatr Soc. 1985; 33(2):116-20.

22.Lipschitz DA. Screening for nutritional status in the elderly. Prim Care. 1994;21(1): 55-67.

23. Ayres M, Ayres Júnior M, Ayres DL, Santos AS. Bioestat 3.0: aplicações estatísticas nas áreas das ciências biológicas e médicas. Brasília: Belém - sociedade civil Mamiraua; 2003.

24.Barkoukis K. Nutrition recommendations in elderly and aging. Med Clin N Am. 2016; 100:1237-1250.

25.Tedrus GMAS, Srebernich SM, Santos TBN. Correlation between clinical and cognitive aspects and nutritional indicators of elderly patients with new-onset epilepsy. Epilepsy Behav. 2018; 85:105-109. 26.Ng TP, Nyunt MSZ, Gao Q, Wee SL, Yap P, Yap KB. Elderly Nutritional Indicators for Geriatric Malnutrition Assessment (ENIGMA): Development and 
validation of a nutritional prognostic index. Clin Nutr ESPEN. 2017; 22:54-63.

27. Borrego CCH, Lopes HCB, Soares MR, Barros VD, Frangella VS. Causas da má nutrição, sarcopenia e fragilidade em idosos. RASBRAN. 2012; 4(5):54-58.

28. Mourão LF, Xavier DAN, Neri AL, Luchesi KF. Association study between natural chronic diseases of aging and swallowing changes referred by community elderly. Audiol Commun Res. 2016; 21:e1657.

Conflito de interesses: Os autores declaram não haver conflito de interesses.

\section{Participação dos autores:}

- Concepção: Aquino TR, Mairink IA, Jesus SC, Cruz GTG, Soares LJF, Souza AF, Martins NRT, Silva VS, Rocha FGS, Prates RP, Alves AMJT, Farias PKS.

- Desenvolvimento: Aquino TR, Mairink IA, Jesus SC, Cruz GTG, Soares LJF, Souza AF, Martins NRT, Silva VS, Rocha FGS, Prates RP, Alves AMJT, Farias PKS.

Redação e revisão: Aquino TR, Mairink IA, Jesus SC, Cruz GTG, Soares LJF, Souza AF, Martins NRT, Silva VS, Rocha FGS, Prates RP, Alves AMJT, Farias PKS.

Como citar este artigo: Aquino TR, Mairink IA, Jesus SC, Cruz GTG, Soares LJF, Souza AF, et al. Avaliação da situação nutricional e dietética de idosos hospitalizados. J Health NPEPS. 2019; 4(2):268279. 